\title{
Formación humanística en estudiantes de educación básica regular
}

\section{Humanistic Training in Students of Regular Basic Education}

\section{Rubí Eloisa Huamán Durand ${ }^{1}$}

Universidad Nacional Mayor de San Marcos | Universidad Continental

rubi.huaman.d@gmail.com

ORCID: https://orcid.org/0000-0003-1114-7676

Citar como: Huamán, R. (2021). Formación humanística en estudiantes de educación básica regular. Desde el Sur, 13(3), e0032

\section{RESUMEN}

La presente investigación parte de la noción de «crisis de las humanidades» propuesta por Martha Nussbaum (2010), quien denuncia la decadencia de la educación a nivel mundial. A partir de ahí, este artículo indaga por el nivel de formación humanística de estudiantes de secundaria de dos colegios peruanos. El diseño utilizado en el estudio fue cuantitativo descriptivo transversal. La muestra se compuso de 350 estudiantes de tercero, cuarto y quinto año de secundaria de educación básica regular de dos centros educativos ubicados en el mismo distrito. Los resultados evidencian que el nivel de formación humanística alcanzado por los estudiantes es, predominantemente, deficiente. Se concluye que urgen estudios que se centren en investigar sobre estrategias didácticas que permitan el desarrollo de la formación humanística en las aulas escolares.

\section{PALABRAS CLAVE}

Crisis de las humanidades, formación humanística, educación básica regular 


\section{ABSTRACT}

This research is based on the notion of «crisis of the humanities» proposed by Martha Nussbaum (2010), who denounces the decline of education worldwide. From there, this article investigates the level of humanistic training of high school students from two Peruvian schools. The design used in the study was quantitative descriptive cross-sectional. The sample consisted of 350 students from the seventh cycle of Regular Basic Education from two educational centers located in the same district. The results show that the level of humanistic training reached by the students is predominantly deficient. It is concluded that studies are urgently focused on investigating teaching strategies that allow the development of humanistic training in school classrooms.

\section{KEYWORDS}

Crisis of the humanities, humanistic training, regular basic education

\section{Introducción}

Hace más de una década, en línea con las investigaciones de Savater (1997) y Culler (1998), la filósofa estadounidense Nussbaum (2010) denunció la presencia de una «crisis silenciosa» en la educación a nivel mundial. La autora alertó que debido a la prioridad de los sistemas educativos en el «utilitarismo» y lo "práctico», ya no se priorizaba el desarrollo humano. La base del problema reside, afirma la investigadora, en la erradicación de los cursos y las carreras artístico-humanistas en todos los niveles educativos por considerarlos infructíferos para el progreso económico personal y nacional, a pesar de que habían demostrado su valor e importancia en la historia de la humanidad. Aunque Nussbaum sustenta su propuesta en casos de Estados Unidos y la India, su análisis es aplicable para los sistemas educativos de todos los países que se encuentran dentro del mercado global. En ellos se aprecia la importancia de las estrategias de la obtención de rentas y los beneficios económicos en las carreras de ciencias, mientras que se silencia y elimina todo lo referente a las artes, las humanidades y el pensamiento crítico.

Luego de la publicación de Nussbaum, otros autores como Steiner (2012), Cortina (2013), Betancour (2021) y Cifuentes (2014) continuaron reflexionando sobre la decadencia de las humanidades y sus efectos en la educación a nivel mundial. En el contexto peruano, el docente sanmarquino Huamán (2012) también ha manifestado su preocupación por cómo se 
han ido eliminando los estudios humanísticos y considera que este declive solo acentúa la crisis educativa mundial y peruana. En esta investigación, consideramos pertinente actualizar la discusión sobre la importancia de la formación humanística en la educación básica regular del Perú, ya que los currículos escolares han atravesado un proceso de «actualización»y, entre los cambios que se han realizado, resalta la reorganización de todos los cursos en solo cuatro áreas curriculares (Comunicación, Personal Social, Psicomotriz y Descubrimiento del Mundo), y algunos cursos como Familia y Civismo, Historia del Perú, Historia Universal, Literatura Peruana, Literatura Española, Filosofía y Lógica se incluyen solo como un «apéndice» o han desaparecido.

Si bien es cierto que "humanismo», «humanidades» y «formación humanística» se encuentran íntimamente relacionados, es indispensable precisar a qué se refieren cada uno de ellos para establecer de manera clara cómo se vinculan. Existen dos ramas del humanismo: la secular y la religiosa (Huitt, 2009)2 ${ }^{2}$. El humanismo secular es heredero de la cultura grecolatina y sostiene que la educación debe ser integradora y totalizante. En esta misma línea, el investigador mexicano López (2000) considera como «humanismo» el marco filosófico que se centra en la actitud «que nace de la convicción profunda en la dignidad y la riqueza del ser humano» (p. 44).

Por su parte, las «humanidades» aluden a los cursos dedicados al estudio de lo humano y, en la actualidad, suelen identificarse con los cursos de «letras» como Historia, Filosofía, Literatura, Psicología, Economía, Geografía, etc. No obstante, es necesario precisar que, tal como afirma Vita (2004), la noción de humanidades es un producto histórico que se fundamenta en la enseñanza de las artes liberales, que se centraban en el estudio de textos humanos. Estas incluían el trivium, encargado del desarrollo de la elocuencia (Gramática, Dialéctica y Retórica) y el cuadrivium, que se concentraba en los números (Aritmética, Geometría, Astronomía y Música). En función de esta armonía de las disciplinas, surge la consideración que sostiene que no deberían existir conflictos entre las humanidades y las ciencias duras. La ruptura del equilibrio renacentista de todas las disciplinas se remonta a los descubrimientos científicos durante la llustración en materias como Física, Química y Biología, lo cual repercutió en la exclusión de las humanidades del campo científico. Esta pugna se trasladó al ámbito educativo, que consideró, de forma errónea, que los contenidos,

2 Los humanistas religiosos basados en el judeocristianismo consideran que la religión juega un papel importante en el desarrollo humano por la salvación del alma humana y, a partir de allí, crearon la oposición cristiano/pagana. 
los métodos y la finalidad de la enseñanza es lo que debía diferenciar las humanidades y las ciencias.

Ahora bien, por formación humanística entendemos el proceso educativo que «tiene como eje fundamental de su quehacer reflexivo, crítico, formativo y pragmático la pregunta acerca del ser del hombre» (López, 2000, p. 44). En ese sentido, contempla el desarrollo de diversas capacidades que varían según la propuesta de los autores (Savater, 1997; López 2000; Peña, 2006; Nussbaum, 2010). No obstante, se pueden sintetizar estas capacidades en las siguientes dimensiones: el análisis crítico, la argumentación, la empatía y la experiencia estética.

El pensamiento crítico se relaciona con la capacidad de trascender la formación memorística y la repetición de ideas para alentar el pensamiento propio. Los investigadores del pensamiento crítico han establecido diversas habilidades para caracterizar esta capacidad (Ennis, 2005; Facione, 2015); sin embargo, nos interesa destacar que no solo se desea desarrollar habilidades cognitivas, sino también una forma de enfrentar a la vida y a la realidad social (Toro et al., 2019). Por ello, implica cuestionar la realidad, defender sus argumentos, analizar realidades y resolver problemas que aquejan a todos.

En relación con lo anterior, encontramos la conciencia histórica que, según López (2000), alude a la comprensión del momento histórico en el que se ubica cada persona, a cómo se considera la herencia de un pasado y la colaboración el futuro. Esta identificación sociohistórica permite considerar la educación como fruto de la herencia cultural de la humanidad y, a partir de allí, adoptar un compromiso sociocultural. Este comprender que somos parte de una sociedad permite el desarrollo de la conciencia social, la cual se caracteriza por priorizar el bien común de la nación. Para ello, es necesario erradicar la educación egoísta e individualista que promueve la competencia en lugar de la empatía, la solidaridad y la capacidad de interacción e integración social. Por último, mencionaremos la necesidad de una auténtica experiencia estética. La comprensión del fenómeno estético se consigue gracias a un continuo contacto con la belleza y la generación de experiencias estéticas. Se relaciona con actitudes y emociones que permitan admirar la belleza y desarrollar la sensibilidad en los estudiantes. López (2000) aclara que va más allá de impartir cursos de arte, ya que incluye la reflexión y apropiación de la ciudad como espacio de talentos.

Por todo lo anterior, el objetivo general del presente estudio responde a la siguiente pregunta de investigación: ¿cuál es el grado de formación humanística de los estudiantes peruanos de tercero, cuarto y quinto año 
de secundaria de educación básica regular? Los objetivos específicos responden a las siguientes preguntas: ¿cuál es el grado de análisis crítico que los estudiantes de tercero, cuarto y quinto año de secundaria de educación básica regular han desarrollado?, ¿cuál es el grado de argumentación que los estudiantes de tercero, cuarto y quinto año de secundaria de educación básica regular han desarrollado?, ¿cuál es el grado de empatía que los estudiantes de tercero, cuarto y quinto año de secundaria de educación básica regular han desarrollado? y ¿cuál es el grado de sensibilidad estética que los estudiantes de tercero, cuarto y quinto año de secundaria de educación básica regular han desarrollado? La hipótesis general que proponemos es que el nivel general de formación humanística y socioemocional de los estudiantes es deficiente.

\section{Materiales y método}

Según los postulados de Hernández, Fernández y Baptista (2014), esta investigación se realizó bajo el enfoque cuantitativo y el alcance descriptivo; ya que se apela a procedimientos estadísticos para recolectar datos, y la finalidad es conocer y detallar las características de un grupo; en este caso, el nivel de formación humanística de los estudiantes. Asimismo, responde a una investigación no experimental, pues no se manipula la variable de estudio, sino que se observa el fenómeno «tal como se da en un contexto natural para analizarlo» (Hernández et al., p. 152). El diseño de investigación responde a un estudio transaccional o transversal, debido a que la recolección de datos se realizó una única vez y en un tiempo específico (Hernández et al.).

\section{Población y muestra}

Nuestra población comprende a los 350 estudiantes de tercero, cuarto y quinto año de secundaria de educación básica regular del turno matutino de dos centros educativos, uno privado y el otro nacional, ambos ubicados en el mismo distrito. En cuanto a la muestra, Hernández, Fernández y Baptista (2014) afirman que debido a que «pocas veces es posible medir a toda la población» se selecciona un subconjunto de esta para que sea «un reflejo fiel del conjunto» (p. 175). No obstante, como en esta investigación sí fue posible medir al total de la población, no se seleccionó un subconjunto del total, sino que se trabajó con el total de la población, la cual presenta la siguiente composición: 
TABLA 1. Composición de la muestra por grado

\begin{tabular}{|c|c|c|}
\hline Grado & Frecuencia & Porcentaje \\
\hline Tercer año & 130 & 37 \\
\hline Cuarto año & 123 & 35 \\
\hline Quinto año & 97 & 28 \\
\hline Total & 350 & 100,0 \\
\hline
\end{tabular}

TABLA 2. Composición de la muestra por edad

\begin{tabular}{lcc} 
& Fiad & Porcentaje \\
14 & 3 & 1 \\
\hline 15 & 109 & 31 \\
\hline 16 & 120 & 34 \\
\hline 17 & 111 & 32 \\
\hline 18 & 7 & 2 \\
\hline Total & 350 & 100,0 \\
\hline
\end{tabular}

TABLA 3. Composición de la muestra por sexo

\begin{tabular}{lll} 
& Fexco & Porcentaje \\
Femenino & 191 & 54 \\
\hline Masculino & 164 & 46 \\
\hline Total & 350 & 100 \\
\hline
\end{tabular}

TABLA 4. Composición de la muestra según tipo de colegio

\begin{tabular}{lcc} 
& Colegio & Precrencia \\
Privado & 95 & 27 \\
\hline Estatal & 255 & 73 \\
\hline Total & 350 & 100 \\
\hline
\end{tabular}

\section{Instrumento}

Con el fin de indagar el nivel de formación humanística de los estudiantes, en este estudio se aplicó un test de 20 preguntas, con formato de respuestas de opción múltiple. Mediante el test se evaluaron cuatro dimensiones: el análisis crítico, es decir, la capacidad de relacionar, discriminar y evaluar diversas ideas; la argumentación, donde se mide la solidez y la justificación de las ideas propuestas; la empatía, que abarca la 
fase emotiva, cognitiva, social; y, por último, la dimensión de la sensibilidad estética (experiencia estética y juicio estético).

El instrumento fue elaborado por la investigadora y fue validado a través de juicio de expertos; en este caso, se recurrió a la opinión de tres docentes investigadores que consideraron que, efectivamente, el instrumento medía la variable formación humanística. Asimismo, para probar la confiabilidad del instrumento, es decir, para medir el grado en el que un instrumento produce resultados consistentes y coherentes (Hernández, 2006), se recurrió a la prueba piloto aplicada a 30 encuestados. Para la medida de consistencia interna, se empleó el alfa de Cronbach y el aplicativo IBM SPSS Statistics 19. El coeficiente obtenido fue 0,85 , que evidencia que las preguntas del test contribuyen de manera significativa a la definición de los conceptos investigados, ya que cuando el coeficiente se aproxima a uno, el instrumento es muy confiable para la investigación.

\section{Procedimiento}

La presente investigación responde al diseño transeccional o transversal descriptivo, por lo que la recolección de datos se realizó en un único momento. La finalidad, como ya se ha mencionado, fue conocer los niveles de formación humanística de los estudiantes de tercero, cuarto y quinto año de secundaria de educación básica regular del turno matutino de dos centros educativos. Por ello, los resultados obtenidos en el trabajo de campo realizado para esta investigación se tabularon y se analizaron con el software IBM SPSS Statistics 19 (Paquete Estadístico para las Ciencias Sociales). A continuación, se presentarán las tablas de frecuencias de cada dimensión de la variable formación humanística. Luego, se efectúa un análisis descriptivo de todos los resultados.

\section{Resultados}

TABLA 5. Dimensión análisis crítico

\begin{tabular}{l|ccc}
\multicolumn{1}{c|}{ Análisis crifico } & Nivel alito & Nirel medio & Nivel bajo \\
\hline Relación de ideas & $61 \%$ & $33 \%$ & $6 \%$ \\
\hline Discriminación de ideas & $76 \%$ & $15 \%$ & $9 \%$ \\
\hline Evaluación de significados & $67 \%$ & $16 \%$ & $17 \%$ \\
\hline
\end{tabular}

En la tabla 5 observamos que los estudiantes del séptimo ciclo de educación básica regular han desarrollado el análisis crítico en la siguiente proporción: la relación ideas se ha alcanzado en 61\%; la discriminación de ideas, en $71 \%$; y la evaluación de significados, en $67 \%$. Estos resultados demuestran que el análisis crítico que presentan los estudiantes oscila 
entre el nivel regular y bueno. Bueno para discriminar ideas, regular para evaluar significado y relacionar ideas.

TABLE 6. Dimensión argumentativa

\begin{tabular}{l|ccc} 
& Nivel alto & Nirel medio & Nivel bajo \\
\hline Solidez argumentativa & $48 \%$ & $34 \%$ & $18 \%$ \\
\hline Justificación & $64 \%$ & $15 \%$ & $21 \%$ \\
\hline Explicación & $73 \%$ & $10 \%$ & $17 \%$ \\
\hline
\end{tabular}

Sobre la argumentación, se aprecia que el $48 \%$ de estudiantes consiguió solidez argumentativa; un $64 \%$ justifica adecuadamente; y un $73 \%$ explica de forma clara. Estos resultados evidencian que la argumentación que presentan los estudiantes oscila entre los tres niveles. Deficiente para identificar respuestas con solidez argumentativa mientras que alto para justificar adecuadamente y para explicar sus posturas sobre un tema polémico.

TABLE 7. Dimensión empatía

\begin{tabular}{llll}
\multicolumn{1}{c}{ Empatia } & Nivel alto & Nivel medio & Nivel bajo \\
Cognitiva & $45 \%$ & $49 \%$ & $6 \%$ \\
\hline Emocional & $33 \%$ & $52 \%$ & $9 \%$ \\
\hline Social & $49 \%$ & $47 \%$ & $4 \%$ \\
\hline
\end{tabular}

Sobre la empatía, los datos arrojan que el $45 \%$ de estudiantes consiguió desarrollar la empatía cognitiva; un 33\% la empatía emocional; y un $49 \%$, la empatía social. Estos resultados demuestran que la empatía que presentan los estudiantes, en sus tres niveles ${ }^{3}$, es mayormente de nivel medio y deficiente.

TABLE 8. Dimensión sensibilidad estética

\begin{tabular}{llll}
\hline Sensibilidad estética & Nivel alto & Nivel medio & Nivel bajo \\
\hline Experiencia estética & $19 \%$ & $57 \%$ & $24 \%$ \\
\hline Juicio estético & $44 \%$ & $46 \%$ & $10 \%$ \\
\hline
\end{tabular}

3 En la investigación se entiende por empatía cognitiva la capacidad de entender cómo piensa el otro y por qué actúa de determinada manera; la empatía emocional alude al respeto y a la solidaridad con los sentimientos del otro; y la empatía social es la capacidad para reflexionar en los problemas sociales a partir de la experiencia individual de otros. 
Acerca de la sensibilidad estética, los datos establecen que el 19\% de estudiantes consiguió la experiencia estética y un $44 \%$, el juicio estético. Estos resultados demuestran que el desarrollo de la sensibilidad estética de los estudiantes es mayormente deficiente.

\section{Discusión y conclusiones}

Los resultados obtenidos en esta investigación son desalentadores. En tres de las cuatro dimensiones analizadas, el nivel de formación humanística es deficiente ${ }^{4}$. La única dimensión que alcanzó un nivel regular y bueno es el referido al "análisis crítico» que se centró en la discriminación y relación de ideas, y evaluación de los significados. Es decir, es una dimensión que se vincula con lo «clásicamente» relacionado con el ámbito académico. De manera similar, la dimensión argumentativa que también se asocia con el campo intelectual obtuvo regular en dos rubros y solo en uno deficiente. En cambio, las dimensiones de empatía y sensibilidad estética que se alejan del ámbito propiamente académico y exploran facetas consideradas más «humanas/humanistas» obtuvieron un nivel deficiente en la mayoría de sus rubros.

Sobre el pensamiento crítico y la argumentación, los resultados coinciden con diversas investigaciones realizadas en el Perú, tanto en estudiantes escolares como universitarios, ya que estos confirman que el nivel de pensamiento crítico oscila entre medio y bajo, y solo en pocos rubros se logra un nivel óptimo (Milla, 2012; Romero, 2012; Perea, 2017; Ríos, 2019; Cangalaya, 2019; Cangalaya, 2020; Sotelo y Espíritu, 2020). Respecto al desarrollo de la argumentación en los estudiantes, es necesario aclarar que algunos autores analizan esta capacidad como un elemento del pensamiento crítico; no obstante, debido a su complejidad, también puede abordarse de manera independiente. En ese sentido, es notorio los vacíos investigativos en el Perú, ya que son escasos los estudios que se centran en describir el nivel de desarrollo de la capacidad argumentativa de los estudiantes y las formas en que se puede mejorarlo. Solo destaca el trabajo de Villanueva (2020), que describe los componentes de la argumentación escolar de estudiantes de quinto año, y la investigación de Hidalgo, Mendoza, Miranda, Navarro, Rodríguez y Salas (2005), que proponen mejorar la capacidad argumentativa de textos orales de estudiantes de segundo grado de secundaria. No obstante, en ambos casos la capacidad argumentativa se circunscribe al campo académico

4 Es necesario resaltar que la investigación se realizó en un colegio nacional y en uno privado. En ambos casos los resultados son similares, por lo que concluimos que la estructura educativa nacional peruana no enfatiza el desarrollo de la formación humanística. 
(textos académicos orales o escritos), excluyendo otros ámbitos que también forman parte del ser humano.

Por lo anterior, reafirmamos la necesidad de investigar sobre prácticas educativas que permitan desarrollar el pensamiento crítico y la argumentación de los estudiantes desde etapas tempranas de la escolaridad. En esta línea, se circunscribe la investigación de Montoya y Monsalve (2008), quienes proponen siete interesantes estrategias didácticas que tienen como punto de partida la realidad de los escolares colombianos de educación básica secundaria. A través del análisis de noticias, medios de comunicación, subculturas, problemas sociales del entorno inmediato, las TIC e imágenes, los autores demostraron que las habilidades del pensamiento crítico y la argumentación de los estudiantes mejoró notablemente. La premisa que guio a Montoya y Monsalve (2008) fue que considerar que ser un ciudadano activo requiere de conocer a profundidad el medio donde nos desarrollamos para «establecer una relación consciente y participativa con el entorno; de no ser así, se cae en el riesgo de la enajenación, la marginación y la masificación consumista, sin hacer uso de la capacidad de pensamiento crítico» (p. 7). Posteriormente, Montoya (2009) aplica una guía para «estimular el proceso de pensamiento crítico» a partir de la reflexión de competencias ciudadanas en estudiantes de secundaria de cuatro centros educativos. En todos los casos, se corroboró que los estudiantes optimizaron sus habilidades de pensamiento crítico y argumentación, y también mejoraron la convivencia y cohesión grupal dentro del entorno escolar. De forma similar, en el Perú destaca la investigación realizada por Miney (2018), que propone utilizar los cuentos clásicos para desarrollar el pensamiento crítico y la argumentación en estudiantes. La autora corroboró que, a través del andamiaje docente y su rol como mediador, se consigue incrementar significativamente las habilidades de los alumnos para realizar comentarios sobre los cuentos y sobre las opiniones de sus compañeros, expresar su acuerdo o desacuerdo con las acciones de los personajes, y plasmar la información del texto a su contexto y a sus experiencias personales.

En cuanto a la empatía, por referirse a la comprensión de lo que otros piensan y sienten, es considerada como uno de los elementos de la vertiente interpersonal de la inteligencia emocional. En ese sentido, se encuentran diversos estudios peruanos que indagan sobre la relación entre la inteligencia emocional y el rendimiento académico, sobre todo en estudiantes universitarios. No obstante, se encuentran pocos estudios que se centran en indagar la forma en que se presenta esta capacidad en las aulas escolares peruanas. Granados (2017) demostró la relación entre 
la empatía cognitiva y las habilidades sociales de los estudiantes de sexto grado de primaria en una institución educativa en Huacho. Los resultados del estudio de la investigadora arrojaron que el $51 \%$ de estudiantes presentaban un nivel malo de empatía; el 17\%, un nivel regular; y un $32 \%$, un nivel bueno; en consonancia con ello, los niveles de habilidades sociales obtenidos fueron $31 \%$ malo, $27 \%$ regular y $42 \%$ bueno. Es decir, se comprobó la relación mutua entre las variables, por lo que se concluyó la necesidad de fomentar y desarrollar la empatía para mejorar las habilidades sociales. Esto último también se demostró en el contexto colombiano, donde Hernández, López y Caro (2017) evidenciaron que mejorar la empatía logra un ambiente escolar libre de violencia. El estudio de Hernández, López y Caro respondía a una investigación acción, es decir, que partía de una problemática institucional. Por ello, en un primer momento, los autores aplicaron un test sobre empatía y los resultados indicaron que los jóvenes tenían empatía cognitiva, es decir, identificaban las emociones de sus compañeros; pero no habían desarrollado la empatía emocional, es decir, «al momento de reaccionar ante los mismos, sus actitudes son de hostigamiento $u$ hostilidad» (227). En un segundo momento, en los cursos de Castellano y Sociales, direccionaron el desarrollo de las clases para fomentar y potenciar la empatía. A través de talleres, análisis de videos y películas sensibilizaron a los estudiantes sobre la empatía y el ambiente escolar. Luego de culminado el ciclo de talleres, se observó una notable disminución de violencia escolar en los estudiantes del aula que fue motivo de intervención.

Como evidencia la investigación de Hernández, López y Caro (2017), no es suficiente desarrollar la empatía cognitiva, ya que esta se limita a reconocer cómo se siente el otro. Es urgente desarrollar la empatía emocional y social, pues nos permiten sentir como el otro y nos motiva a ayudarlo. Estos dos últimos tipos de empatía resultan ser más importantes, ya que, como mencionan Muñoz y Chaves (2013), la empatía es considerada como una competencia ciudadana que influencia en la conducta prosocial solo si «motiva a las personas a tener comportamientos de ayuda, cuidado, evitar el daño a otro, buscar conciliar diferencias y pedir perdón cuando se reconocen equívocos; aumenta la independencia en la toma de decisiones y mejora la salud mental» (134). La necesidad de desarrollar la empatía en sus tres dimensiones es imprescindible en el momento actual, cuando prima el individualismo y la competitividad.

Por último, la sensibilidad estética de los estudiantes peruanos de educación básica regular debe ser declarada en emergencia, ya que la esencia del humanismo es la "cultura del alma», es decir, "o cultivo desinteressado do espírito e do gosto; da capacidade de fruir, apreciar 
e julgar as instituições e obras que integram nosso mundo comum» (Fonseca, 2017, p. 1033). Actualmente, las materias relacionadas con el arte son consideradas como poco productivas, e incluso se las tilda de «relleno» en el currículo. Las razones por las que estas son consideradas negativamente responden a la poca utilidad en el mundo actual, pues «no se vive del arte», pero también a la forma en cómo esta es desarrollada en las aulas escolares. Si el curso de arte se reduce a dibujar y a pintar sin un fin específico, pierde todo el potencial que puede ser utilizado para mejorar la formación de los estudiantes. Arbocó (2020) advierte que uno de los errores de la educación es considerar solo la dimensión intelectual en el ámbito educativo y desestimar lo emocional y lo ético. Asimismo, el autor afirma que la escuela no debe ser un espacio donde solo se transmiten conocimientos que garanticen el ingreso a la universidad, sino que es necesario promover el arte dentro de las escuelas por los diversos beneficios que este tiene. Por ejemplo, permite que los jóvenes encuentren sentido a sus vidas, controlar sus miedos y frustraciones, y, de igual forma, estimula su creatividad, refuerza su autoestima y se convierte en un alivio al estrés escolar. Asimismo, prosigue el autor, las diferentes manifestaciones del arte le brindarán al estudiante experiencias que podrá utilizar en su vida cotidiana durante toda su existencia, como saber diferenciar «lo estético de lo grotesco», comprender mejor las emociones humanas y no dejarse arrastrar por seudoarte pasajero. En síntesis, «puede fomentar en el estudiantado un espíritu crítico, una actitud más contemplativa, un sentido ético e inclusivo que de pronto aún les está costando conseguir, y es que las ciencias puras y exactas todavía no pueden decirnos lo que es bello, justo y humano. Y el arte sí que puede, o al menos lo intenta» (p. 17).

La situación actual de la formación humanística en las aulas escolares peruanas puede entenderse mejor gracias al estudio de Sánchez (2014). El autor analizó las percepciones de los docentes de educación básica regular sobre la educación humanista y las prácticas pedagógicas que buscan concretarla. Entre los resultados de la investigación, destaca que el $45 \%$ de los encuestados considera que se deben desarrollar todas las dimensiones de la educación humanista; pero el $41 \%$ creen necesario solo centrarse en las habilidades intelectuales. Estos datos nos revelan que son los propios docentes los que le restan valor a las dimensiones «no intelectuales» de la formación humanística de los estudiantes y, en consecuencia, es más probable que centran sus prácticas en desarrollar solo «lo intelectual». En ese sentido, es necesario sensibilizar a los docentes sobre lo que implica la humanización y la importancia de desarrollar todas las áreas de la formación humanística, ya que considerar la educación 
como solo transmisora de conocimientos es tener una visión sesgada de la finalidad de la educación y de la formación humanística.

Por todo lo anterior, consideramos que es indispensable repensar diversas aristas relacionadas con la formación humanística de los estudiantes de educación básica. Sin embargo, en cuanto a las limitaciones del estudio, cabe señalar la necesidad de explorar en mayor profundidad las implicancias de la metodología docente en el desarrollo de la formación humanística a través de investigaciones experimentales. En definitiva, este estudio es un punto de partida para otras iniciativas orientadas a conocer con mayor profundidad cómo incrementar la formación humanística, ya que es necesario que los estudiantes desarrollen capacidades que enriquezcan al ser humano.

\section{Contribución de autoría}

Rubí Eloisa Huamán Durand es autora del artículo y responsable de la estructura y redacción del documento.

\section{Fuente de financiamiento}

Autofinanciado.

\section{Potenciales conflictos de interés}

Ninguno. 


\section{REFERENCIAS BIBLIOGRÁFICAS}

Arbocó, M. (2020) Para que aflore lo mejor de nosotros. Educación, 26(1), 11-28. https://revistas.unife.edu.pe/index.php/educacion/article/ view/2180/2247

Betancur, M. (2021). La democracia necesita de las humanidades. Revista Aleph, 55(196), 121-141. https://www.revistaaleph.com.co/images/ ediciones_pdf/Revista_Aleph_196.pdf\#page $=123$

Cangalaya, L. (2019). Habilidades comunicativas y pensamiento crítico en estudiantes de primer año del curso de Introducción a la Literatura, Facultad de Letras y Ciencias Humanas, de la Universidad Nacional Mayor de San Marcos, 2017. [Tesis de doctorado, Universidad Nacional Mayor de San Marcos].

Cangalaya, L. (2020). Habilidades del pensamiento crítico en estudiantes universitarios a través de la investigación. Desde el Sur, 12(1), 141153. https://revistas.cientifica.edu.pe/index.php/desdeelsur/article/ view/595/637

Cifuentes, J (2014). El papel de las humanidades en la educación superior en el siglo XXI. Revista Quaestiones Disputatae, (15), 101-112. http:// revistas.ustatunja.edu.co/index.php/qdisputatae/article/view/842/816

Cortina, A. (2013). El futuro de las humanidades (una preocupación internacional). Revista Chilena de Literatura, (84) https://scielo.conicyt.cl/ scielo.php?pid=S0718-22952013000200015\&script=sci_arttext

Culler, J. (1998). El futuro de las humanidades. En E. Sullá (Ed.), El canon literario (pp. 139-160). Arco/Libros.

Ennis, R. (2005). Pensamiento crítico: un punto de vista racional. Revista de Psicología y Educación, (1), 47-64.

Facione, P. (2015). Critical thinking: What it is and why it counts. Measured Reasons LLC.

Fonseca, J. (2017). Os ideais da formação humanista e o sentido da experiência escolar. Educação e Pesquisa, 43(4), 1023-1034. https://www. scielo.br/j/ep/a/ZVMwkbKyWZB4GbjDBmQRKzp/?lang=pt

Granados, A. (2017). La empatía cognitiva y las habilidades sociales de los estudiantes de sexto grado de primaria de la institución educativa Santa Rosa - Huacho, 2017. [Tesis de maestría, Universidad César Vallejo].

Hernández, J., López, R. y Caro, O. (2018). Desarrollo de la empatía para mejorar el ambiente escolar. Educación y Ciencia, 21, 217-244. https://revistas.uptc.edu.co/index.php/educacion_y_ciencia/article/ view/9407/7854 
Hernández, R., Fernández, C. y Baptista, P. (2014). Metodología de la Investigación. (6. ${ }^{\mathrm{a}}$ ed.). McGrall Hill.

Hidalgo, V., Mendoza, Y., Miranda, E., Navarro, V., Rodríguez, Z. y Salas, W. (2005). El taller «Comparte tu pensar en voz alta» basado en la teoría de la argumentación de Stephen Toulmin y la teoría social por imitación de Albert Bandura eleva el nivel de argumentación de textos orales en los alumnos de 2.do B de secundaria del colegio Sagrado Corazón "Chalet» del distrito de Chorrillos perteneciente a la UGEL 07. [Tesis de licenciatura, Instituto Pedagógico Nacional Monterrico].

Huamán, A. (2012). La formación humanista: fundamentos y desafíos. Fondo Editorial de la Universidad Científica del Sur.

Huitt, W. (2009). Humanismo y educación abierta. Psicología Educativa Interactiva. Universidad Estatal de Valdosta. http://www.edpsycinteractive. org/topics/affect/humed.html

López, M. (2000). Desarrollo humano y práctica docente. Trillas.

Milla, M. (2012). Pensamiento crítico en estudiantes de quinto de secundaria de los colegios de Carmen de la Legua Callao. [Tesis de maestría, Universidad San Ignacio de Loyola].

Miney, K. (2018). La iniciación al pensamiento crítico a través del uso de cuentos clásicos. [Tesis de licenciatura, Pontificia Universidad Católica del Perú].

Montoya, J. y Monsalve, J. (2008). Estrategias didácticas para fomentar el pensamiento crítico en el aula. Revista Virtual Universidad Católica del Norte, 25. https://www.redalyc.org/pdf/1942/194215513012.pdf

Montoya, J. (2009). Guía metodológica para el fomento de las competencias ciudadanas en la básica secundaria a partir del pensamiento crítico. Revista Virtual Universidad Católica del Norte, 29. https://www.redalyc.org/ pdf/1942/194214466002.pdf

Muñoz, A. y Chaves, L. (2013). La empatía: ¿un concepto unívoco? Kaharsis, 16, 123-143. http://bibliotecadigital.iue.edu.co/bitstre a m/20.500.12717/2391/1/467-Texto\%20del\%20art\%c3\%adcu lo-4961-1-10-20200913.pdf

Nussbaum, M. (2010). Sin fines de lucro. Por qué la democracia necesita de las humanidades. Katz.

Peña, V. (2006). ¿Por qué es importante la formación humanística? La Educación Superior en crisis. Letras (77), 111-112, 169-174.

Perea, L. (2017). Disposición hacia el pensamiento crítico y su relación con el rendimiento académico en estudiantes del curso de metodología de la investigación de una universidad privada de Lima Metropolitana. [Tesis de maestría, Universidad Peruana Cayetano Heredia]. 
Ríos, Y. (2019). Pensamiento crítico y comprensión de lectura en estudiantes de cuarto año de secundaria de un colegio particular del distrito de San Isidro. [Tesis de maestría, Universidad Ricardo Palma].

Romero, F. (2012). Influencia del programa «DPC», en el desarrollo del pensamiento crítico, en el área de ciencias sociales, en los estudiantes del segundo grado de secundaria de la I. E. N. ${ }^{\circ} 81003$ "César Abraham Vallejo Mendoza», Trujillo, 2008. [Tesis de maestría, Universidad César Vallejo].

Sánchez, A. (2014). Percepciones de docentes sobre la Educación Humanista y sus dimensiones. Revista Educativa Hekademos, 17, 7-22. http://www.hekademos.com/hekademos/media/articulos/17/01.pdf

Savater, F. (1997). ¿Hacia una humanidad sin humanidades? En El valor de educar. (2. ${ }^{\text {a ed.) }}$ (pp. 50-62). Ariel.

Sotelo, R. y Espíritu, J. (2020). Formación general humanista en universidades públicas. Revista Ciencias y Humanidades, 10 (10), 277-295.

Steiner, G. (2012). Humanities-at twilight? En R. Gil Soeiro y S. Tavares (Eds.), Rethinking the humanities. Paths and challenges (pp. 25-41). Cambridge Scholars Publishing.

Toro, E. Ponce, R., Ramírez, R. y Navia, J. (2019) Pensamiento críticocomplejo-innovador: reencuentro con una pedagogía. Revista Dilemas Contemporáneos: Educación, Política y Valores, 1-16.

Villanueva, M. (2020). La argumentación en la esfera escolar: Análisis pragmalingüístico. [Tesis de maestría, Universidad Nacional Mayor de San Marcos].

Vita, S. (2004). Sobre el problema de la enseñanza de las humanidades en la Educación Secundaria. Didáctica. Lengua y Literatura, 16, 251-269. https:// revistas.ucm.es/index.php/DIDA/article/view/DIDA0404110251A/19375 